\title{
METODOLOGÍA PARA LA TIPIFICACIÓN HIDROMORFOLÓGICA DE LOS CURSOS FLUVIALES DE ARAGÓN EN APLICACIÓN DE LA DIRECTIVA MARCO DE AGUAS (2000/60/CE)
}

\author{
Alfredo Ollero Ojeda, $\mathrm{M}^{\mathrm{a}}$ Teresa Echeverría Arnedo, Miguel Sánchez Fabre, \\ Virginia Auría Izquierdo, Daniel Ballarín Ferrer y Daniel Mora Mur \\ Dpto. de Geografía y Ordenación del Territorio, Universidad de Zaragoza \\ Pedro Cerbuna, s/n. 50009 Zaragoza. e-mail: aollero@unizar.es
}

\begin{abstract}
Resumen: Diferenciar distintos tipos de cursos de agua en la red fluvial es un proceso clave de la aplicación de la Directiva 2000/60/CE a la determinación de la calidad ecológica de los ríos. Se aporta un sencillo método de tipificación basado en criterios hidromorfológicos y apoyado en los descriptores que propone la Directiva. Se establecen tres grandes grupos de criterios de tipificación: topográficos, hidroclimáticos y geomorfológicos. A continuación se desarrolla un proceso de simplificación que desemboca en la definición de 13 tipos básicos aplicables a la red fluvial aragonesa.
\end{abstract}

Palabras clave: sistemas fluviales, variables hidromorfológicas, corredores ribereños, Directiva 2000/60/CE, Aragón.

\begin{abstract}
Classification of the different types of rivers and streams is a key process in the application of the Directive 2000/60/EC to determine the ecological quality of rivers. We report a simple method of characterization based on hydromorphological standards and on the description criteria proposed by the Directive. Three overall groups of characterization criteria are established: topographical, hydroclimatic and geomorphological. After that a simplification process is performed, leading to the definition of 13 basic types applicable to the Aragonese drainage network.
\end{abstract}

Key words: fluvial systems, hydromorphological features, riparian corridors, Water Framework Directive, Aragon.

\footnotetext{
* Recibido: 28-01-2003. Aceptado: 30-04-2003.
} 


\section{Introducción}

Los ríos son sistemas naturales enormemente dinámicos y complejos. Su principal función es el transporte de agua, sedimentos y nutrientes, pero además conforman espacios lineales de gran valor ecológico, paisajístico y territorial, auténticos corredores que enlazan montañas y tierras bajas. Por tanto, la red fluvial, los ríos o sistemas fluviales, constituye un elemento clave en la dinámica ambiental y en la planificación territorial.

La comunidad autónoma de Aragón se localiza en el Nordeste de la Península Ibérica. Se trata de un territorio de interior y con notables contrastes climáticos y altitudinales, en función de tres grandes unidades de relieve: Pirineos al Norte, Depresión del Ebro en el centro y Cordillera Ibérica al sur. La aridez del sector central hace que los sistemas fluviales adquieran un notable papel en el territorio, siendo muy compleja la gestión del agua en la región.

La tipificación de los sistemas fluviales de un territorio es un paso previo imprescindible para la posterior determinación de su estado ecológico. Consiste en tipificar, clasificar y caracterizar para aportar una información clave de cada uno de los tramos de la red fluvial aragonesa, así como para fijar los puntos de muestreo de los distintos parámetros, definir los tramos representativos y establecer las condiciones de referencia especificas de cada tipo.

Teniendo en cuenta la complejidad del funcionamiento dinámico e integrado de los sistemas fluviales desde una perspectiva hidrológica, geomorfológica y ecológica, se ha considerado conveniente utilizar como criterios de tipificación diversos parámetros que constituyen a su vez indicadores de dicho funcionamiento y que están recogidos en el anexo II de la Directiva 2000/60/CE.

La tipificación ha puesto de manifiesto, ante todo, la enorme diversidad de los sistemas fluviales aragoneses, diversidad tanto ecológica (ecodiversidad) como paisajística, así como más estrictamente biótica (biodiversidad) y geomorfológica. En suma, los sistemas fluviales de Aragón constituyen un patrimonio natural de primer orden, y con la aplicación de la Directiva puede avanzarse en su mejora ambiental y en su conservación como espacio natural.

\section{Antecedentes para el diseño metodológico}

No existe en la actualidad una metodología en el trabajo de tipificación aceptada unánimemente o recomendada por la propia Comisión Europea. De hecho, las úni- 
cas recomendaciones publicadas hasta el momento son las del documento de la Comisión Guidance standard CEN TC 230/WG 2/TG 5: N32 (mayo de 2002) y las del grupo de trabajo 2.3 REFCOND, que lanzó en julio de 2002 su primera versión del Guidance on establishing reference conditions and ecological status class boundaries for inland surface waters (Wallin et al., 2002).

Sin embargo, la tradición en el estudio de los sistemas fluviales es larga y fructífera, constituyendo uno de los temas más tratados por las ciencias ambientales. Centrándonos en los antecedentes más directos, en los que en mayor medida nos podemos apoyar para crear una metodología actualizada y adaptada, no se pueden olvidar los innumerables intentos clasificatorios de cursos fluviales desarrollados sobre criterios geomorfológicos. Así, son clásicas la clasificación de Leopold y Wolman (1957) -cauces rectos, trenzados y meandriformes- y la tipificación de Schumm $(1963,1977)$, basada en la estabilidad del canal (estables, que erosionan o que depositan), la carga sedimentaria (de fondo, en suspensión o mixta) y las dimensiones del canal. Complejas pero muy interesantes y aplicables clasificaciones a partir de fotografía aérea se llevaron a cabo en Canadá por Kellerhals et al. (1972, 1976), Galay et al. (1973) y Mollard (1973). Otras más recientes son las descriptivas de Brice y Blodgett (1978), Church y Rood (1983), Mosley (1987), Nanson y Croke (1992) o Downs (1995). Una de las clasificaciones más completas y aplicables a la ordenación es la que propone Rosgen (1994), que combina criterios hidrológicos, geomorfológicos y ecológicos.

En los últimos años el interés por tipificar y clasificar ha dado un salto desde el ámbito científico al técnico, ya que en la mayoría de los países desarrollados han aparecido planes de ordenación, programas de conservación o normativas diversas que afectan a cauces y riberas. Como los sistemas fluviales son enormemente diversos entre sí, era necesario simplificar esta realidad estableciendo tipos, para posteriormente aplicar a cada tipo un modelo de gestión o de actuación. Entre las numerosas iniciativas científico-técnicas que han tratado de tipificar-clasificar cursos fluviales podemos destacar las norteamericanas "River Habitat Survey" (RHS), "System for Evaluating Rivers for Conservation" (SERCON) o "Rapid Stream Assessment Technique" (RSAT), la inglesa "Stream reconnaissance" (Thorne, 1998) o la francesa "SEQ-Physique" (Système d'Evaluation de la Qualité Physique des Cours d'Eau), propugnada por las Agences d'Eau pero con resultados muy discutidos (Tartar, 2001). La más interesante y aprovechable, desde nuestro punto de vista, es la iniciativa australiana del "River Styles Framework" (Brierley y Fryirs, 2000; Fryirs y Brierley, 2001; Brierley et al., 2002), herramienta con base geomorfológica desarrollada en el Dpt. of Physical Geography de la Macquaire University.

Paralelamente a estas propuestas hay numerosas iniciativas que aportan criterios o métodos de valoración interesantes, como por ejemplo los distintos índices de cali- 
dad de espacios ribereños: QBR (Qualitat del Bosc de Ribera), QHEI (Qualitative Habitat Evaluation Index), RCE (Riparian, Channel and Environmental Inventory) o ISC (Index of Stream Condition), que incluyen criterios hidromorfológicos. Por otro lado, en España existe un antecedente de trabajo: la tipificación propuesta por Prat y Munné (1999) para la cuenca del Ebro.

\section{Criterios de tipificación}

De acuerdo con la Directiva 2000/60/CE, la tipificación consiste en clasificar una red hidrográfica en tipos de ríos en función de diversos criterios y variables, con objeto de diseñar la red de seguimiento del estado ecológico y fijar las condiciones de referencia.

Un primer paso consiste en fijar los criterios de tipificación. Para ello hay, por un lado, que respetar los que recoge la Directiva (en los sistemas A y B de su anexo II) y por otro, aprovechando la capacidad de maniobra que la Directiva permite, utilizar algunos parámetros que son indicadores del complejo funcionamiento dinámico e integrado de los sistemas fluviales desde una perspectiva hidrológica, geomorfológica y ecológica. Desde nuestra experiencia, la tipificación debe basarse fundamentalmente en criterios hidromorfológicos, máxime teniendo en cuenta que posteriormente lo que se va a valorar es el estado ecológico a partir de indicadores fundamentalmente biológicos.

Así pues, fue preciso analizar en primera instancia los descriptores obligatorios y optativos de los sistemas A y B del apartado 1.2.1 del Anexo II de la Directiva 2000/60/CE, así como otros parámetros no recogidos en la misma, procediéndose a una progresiva selección de criterios de tipificación. Igualmente se atendió a las recomendaciones emanadas del documento Guidance standard CEN TC 230/WG 2/TG 5: N32.

\subsection{Los sistemas A y B del anexo II de la Directiva 2000/60/CE como base de tipificación}

El objetivo de los descriptores que se incluyen en los sistemas A y B del anexo II es garantizar que se puedan derivar con fiabilidad las condiciones biológicas de referencia de cada uno de los tipos de río. La Directiva señala en concreto lo que se expone en el cuadro 1. 


\section{Cuadro 1. Sistemas A y B del anexo II de la Directiva 2000/60/CE.}

Los Estados miembros determinarán la situación y los límites de las masas de agua superficial y llevarán a cabo una caracterización inicial de dichas masas de agua de conformidad con la siguiente metodología. Los Estados miembros podrán agrupar distintas masas de agua superficial a efectos de dicha caracterización inicial.

I) Las masas de agua superficial dentro de la demarcación hidrográfica se clasificarán en uno de los siguientes tipos de aguas superficiales -ríos, lagos, aguas de transición, aguas costeras- o como masa de agua superficial artificial o como masas de agua superficial muy modificadas;

II) Para cada categoria de aguas superficiales, las masas pertinentes de aguas superficiales de la clemarcación hidrográfica se clasificarăn por tipos. Estos tipos son los que se definen utilizando "el sistema A" o "el sistema B" descritos en la sección 1.2;

III) Si se utiliza el sistema A, se clasificarán primero las masas de agua superficial de la demarcación hidrográfica en las regiones ecológicas correspondientes de conformidad con las zonas geográficas descritas en la sección 1.2 y que figuran en el mapa correspondiente en el anexo XI. A continuación, se clasificarán las masas de agua de cada región ecológica en tipos de masas de aguas superficiales según los descriptores establecidos en los cuadros correspondientes al sistema A;

IV) Si se utiliza el sistema B, los Estados miembros deben lograr, por lo menos, el mismo grado de discriminación que se lograría con el sistema A. En consecuencia, se clasificarán las masas de aguas superficiales de la demarcación hidrográfica en tipos utilizando los valores correspondientes a los descriptores obligatorios y a los descriptores optativos, o combinaciones de descriptores, que se requieran para garantizar que se puedan derivar con fiabilidad las condiciones biológicas de referencia específicas del tipo;

V) Para las masas de agua superficial artificiales y muy modificadas, la clasificación se llevará a cabo de conformidad con los descriptores correspondientes a cualquiera de las categorias de aguas superficiales que más se parezca a la masa de agua muy modificada o artificial de que se trate;

V) Los Estados miembros facilitarán a la Comisión un mapa, o mapas, (en formato SIG) de la situación geográfica de los tipos coherente con el grado de discriminación requerido en el sistema A.

Para "ríos" (apartado 1.2.1. del anexo II) se plantean los siguientes descriptores en el sistema A (cuadro 2).

En relación con la aplicación de este sistema A es preciso exponer las siguientes consideraciones de partida:

- El territorio aragonés se divide entre dos regiones ecológicas: Región Ibéricomacaronésica (1) y Pirinéos (2).

- Parece entenderse que la tipología en función de la altitud se refiere a la cota de altitud de la masa de agua, es decir, del cauce fluvial.

- Parecen despreciarse las cuencas vertientes inferiores a $10 \mathrm{~km}^{2}$, por lo que, según este sistema, se desestiman los tramos fluviales de cabecera y los afluentes de corto recorrido. 
Cuadro 2. Descriptores del sistema A.

\begin{tabular}{|l|c|}
\hline Tipología fijada & Descriptores \\
\hline Región ecológica & Regiones ecológicas que figuran en el mapa A del anexo XI \\
\hline Tipo & $\begin{array}{c}\text { Tipología en función de la altitud } \\
\text { alto }>800 \mathrm{~m} \\
\text { altura media } 200 \text { a } 800 \mathrm{~m} \\
\text { tierras bajas }<200 \mathrm{~m}\end{array}$ \\
& $\begin{array}{l}\text { - Tipología según el tamaño en función de la superficie de la cuenca de alimentación } \\
\text { pequeño } 10-100 \mathrm{~km}^{2} \\
\text { mediano }>100 \text { a } 1000 \mathrm{~km}^{2} \\
\text { grande }>1000 \text { a } 10000 \mathrm{~km}^{2} \\
\text { muy grande }>10000 \mathrm{~km}^{2}\end{array}$ \\
& $\begin{array}{c}\text { Geología } \\
\text { calcáreo } \\
\text { siliceo } \\
\text { orgánico }\end{array}$ \\
\hline
\end{tabular}

Fuente: Directiva 2000/60/CE, anexo II

- En Geología el tipo "orgánico" no tiene sentido en el territorio aragonés. Habría, en cambio, que incluir tipos adicionales: "evaporítico", "detrítico", "mixto"...

Los descriptores o parámetros del sistema $\mathrm{B}$ se recogen en el cuadro 3.

\section{Cuadro 3. Descriptores del sistema B.}

\begin{tabular}{|l|l|}
\hline Caracterización alternativa & $\begin{array}{l}\text { Factores físicos y químicos que determinan las características del río o parte } \\
\text { del río y, por ende, la estructura y composición de la comunidad biológica }\end{array}$ \\
\hline Factores obligatorios & $\begin{array}{l}\text { altitud } \\
\text { latitudi } \\
\text { longitud } \\
\text { geología } \\
\text { tamaño }\end{array}$ \\
\hline Factores optativos & $\begin{array}{l}\text { distancia desde el nacimiento del río } \\
\text { energía de flujo (función del caudal y de la pendiente) } \\
\text { anchura media del agua } \\
\text { profundidad media del agua } \\
\text { pendiente media del agua } \\
\text { forma y configuración del cauce principal } \\
\text { categoría según la aportación fluvial (caudal) } \\
\text { forma del valle } \\
\text { transporte de sólidos } \\
\text { capacidad de neutralización de ácidos } \\
\text { composición media del sustrato } \\
\text { cloruros } \\
\text { oscilación de la temperatura del aire } \\
\text { temperatura media del aire } \\
\text { precipitaciones }\end{array}$ \\
\hline
\end{tabular}

Fuente: Directiva 2000/60/CE, anexo II 
En relación con la aplicación de este sistema B hay varias cuestiones a definir 0 aclarar:

- Los factores obligatorios reproducen básicamente los descriptores del sistema A. Altitud, latitud y longitud pueden entenderse como coordenadas de localización, por lo que se georreferenciarían a los puntos de inicio y final de cada tramo fluvial. El factor geologia puede aplicarse del mismo modo que en el sistema A o bien establecer una escala más diversa que permita definir mejor los caracteres geológicos de la cuenca vertiente. El factor "tamaño" se refiere a la superficie de la cuenca vertiente en cada curso o tramo de aplicación.

- Los factores optativos, como su propio nombre indica, requieren una selección interna, por lo que algunos de ellos pueden ser desechados. Se definen a continuación uno por uno:

Distancia desde el nacimiento del río: es un parámetro básico que hay que referir a los puntos inicial y final de cada tramo. No obstante, es un parámetro caracterizador pero no tipificador, ya que pierde relevancia en el momento en que se dividen los cursos de agua en tramos o sectores funcionales internamente homogéneos.

Energía de flujo (función del caudal y de la pendiente): puede emplearse el parámetro "potencia específica en bankfull" $(\Omega)$, que se obtiene (en vatios $/ \mathrm{m}^{2}$ ) de acuerdo con la fórmula: $\Omega=\rho \mathrm{g} \mathrm{Q}_{\mathrm{b}} \mathrm{S} / \mathrm{w}$, siendo $\rho$ la masa volumétrica del agua $\left(1.000 \mathrm{~kg} / \mathrm{m}^{3}\right)$, g la aceleración de la gravedad $\left(9,8 \mathrm{~m} / \mathrm{s}^{2}\right), \mathrm{Q}_{\mathrm{b}}$ el caudal bankfull $\left(\mathrm{m}^{3} / \mathrm{s}\right)$, $\mathrm{S}$ la pendiente local $(\mathrm{m} / \mathrm{m})$ y w la anchura bankfull $(\mathrm{m})$.

Anchura media del agua: no puede referirse al caudal circulante puntual (en el momento de observación), sino que habrá que trabajar con la situación de bankfull (Williams, 1978), estimando la anchura media que alcanza en cada tramo.

Profundidad media del agua: del mismo modo que la anchura, hay que referirla a la situación bankfull y establecer un valor medio para el tramo.

Pendiente media del agua: se calculará el desnivel y la pendiente media del tramo.

Forma y configuración del cauce principal: se refiere a la geomorfología del cauce, factor caracterizador fundamental, y puede resolverse mediante clásicas clasificaciones de tipos o estilos fluviales apoyadas en la sinuosidad y en la subdivisión o no de canales.

Categoría según la aportación fluvial (caudal): el caudal medio real o estimado es otro parámetro clasificatorio básico. No obstante, probablemente sean más útiles para tipificar el caudal específico y el tipo de régimen hidrológico. 
Forma del valle: carácter morfológico que condiciona en mayor medida a la anchura y desarrollo del corredor ribereño que a la masa de agua. Introduce un valor más paisajístico que funcional, por lo que puede considerarse un factor descriptor secundario.

Transporte de sólidos: es una de las funciones básicas de los sistemas fluviales, aunque es difícil de evaluar. Una posibilidad sería medir en cada tramo el centilo o bloque transportado más grueso, pero como indicador daría, teóricamente, un resultado equivalente al cálculo de la potencia específica del flujo en bankfull. Otras posibilidades más costosas consistirían en realizar análisis granulométricos y morfométricos de sedimentos, o bien tomar muestras de materiales en suspensión en cada punto.

Capacidad de neutralización de ácidos: básicamente puede entenderse como la cantidad de ácido que puede absorber el agua sin cambiar su pH, y se mediría en laboratorio a partir de muestras de campo.

Composición media del sustrato: granulometrías dominantes en el lecho del cauce. Su análisis requiere trabajo de campo y una definición previa del nivel de detalle.

Cloruros: concentración de cloruros en el agua, igualmente se mediría en laboratorio a partir de muestras de campo.

Oscilación de la temperatura del aire: puede referirse a la oscilación térmica anual o a la diaria. Puede ser un parámetro interesante para el estado de los seres vivos acuáticos, pero se carece de datos suficientes para aplicarlo a cada tramo fluvial y no aportaría nada el tomar medidas puntuales en el trabajo de campo.

Temperatura media del aire: se plantea la duda de si se refiere a la de la cuenca o a la del tramo o punto de muestreo. A escala de Aragón no parece un indicador interesante, ya que el principal condicionante es la altitud, parámetro ya recogido más arriba.

Precipitaciones: la pluviometría sobre la masa de agua o en la cuenca no se considera un parámetro interesante ni fácil de evaluar, ya que puede ser mejor representado por el propio caudal, absoluto o específico.

\subsection{Selección de criterios de tipificación}

Los trabajos de tipificación deben servir de apoyo a la valoración de los diferentes indicadores que lleven a la determinación del estado ecológico de los ríos de una región o cuenca. Dado que el objetivo final es esa evaluación del estado ecológico, es fundamental establecer a priori cuáles son los caracteres que definen o aportan 
una valoración alta de dicho estado ecológico en un sistema fluvial. Desde nuestro punto de vista y experiencia, esos caracteres son los siguientes: continuidad, naturalidad, complejidad y dinámica, que definen la funcionalidad como sistema hidromorfológico y ecológico, más singularidad u originalidad en el contexto aragonés y europeo.

Atendiendo a los valores citados, es preciso desarrollar una profunda reflexión sobre los criterios y descriptores más indicados para la tipificación, ya que resulta inviable apoyarse en todos. De hecho, para su efectividad y utilidad consideramos que de la tipificación no deberían resultar más de una decena o una quincena de tipos, sin descartar la posibilidad de diferenciar subtipos a posteriori para determinados objetivos concretos.

Si se aplicara estrictamente el sistema A, o su equivalente los factores obligatorios del sistema $\mathrm{B}$, el resultado de combinar los diferentes criterios daría lugar como mínimo a una tipificación en 96 tipos, ya que en Aragón se dan dos regiones ecológicas, cursos fluviales en los tres niveles de altitud $(>800 \mathrm{~m}, 200-800 \mathrm{~m},<200 \mathrm{~m})$, cuencas de todos los tamaños $\left(<100 \mathrm{~km}^{2}, 100-1.000 \mathrm{~km}^{2}, 1.000-10.000 \mathrm{~km}^{2}\right.$ y $>10.000$ $\mathrm{km}^{2}$ ) y con complejas litologías (geología calcárea o silícea, pero también al menos detrítica y mixta).

Resultan demasiados tipos - una prueba más de la diversidad de Aragón-y en la mayoría de los casos no quedarían bien definidos. No parece recomendable, por tanto, ceñirse a estos criterios de forma estricta, ya que no parecen muy apropiados para el territorio aragonés, bien porque han sido diseñados a partir de ejemplos de otros países europeos, bien porque se ha pensado en su aplicación a escala de grandes estados. Parece imprescindible desechar algunos criterios y, sobre todo, tomar nuevos criterios desde los factores optativos del sistema B. Ahora bien, tal como se ha señalado, éstos presentan en algunos casos problemas de ambigüedad y de solapamiento entre sí.

Sintetizando, todos los factores del sistema B (tanto obligatorios como optativos) pueden ordenarse en tres grandes grupos:

a) Factores topográficos: altitud, latitud, longitud, tamaño de cuenca, distancia desde el nacimiento, pendiente.

b) Factores hidroclimáticos: caudal, temperatura, precipitaciones, capacidad de neutralización de ácidos, cloruros.

c) Factores geomorfológicos: geología, energía del flujo, anchura, profundidad, morfología del cauce y del valle, transporte de sólidos, composición media del sustrato. 


\subsubsection{Factores topográficos}

Entre ellos la altitud es el más importante, ya que ofrece una gradación ecológica y está relacionado con el perfil longitudinal de los ríos. Sin embargo, requiere una adaptación al territorio aragonés:

- Por debajo de la cota $200 \mathrm{~m}$, fijada en la Directiva, hay muy pocos cursos fluviales y no son diferentes de otros tramos por encima de dicha cota; por tanto, hemos decidido no tenerla en cuenta en la tipificación.

- En cambio, la cota $800 \mathrm{~m}$ puede y debe ser mantenida como criterio interesante, ya que en Aragón viene a separar bastante bien los cursos de las áreas montañosas pirenaica e ibérica respecto de aquellos de los somontanos y zona central de la Depresión del Ebro.

- Teniendo en cuenta las altitudes elevadas que se alcanzan en nuestra comunidad, se ha creído conveniente añadir otra cota que separara la media montaña de la alta montaña. La más adecuada ha resultado ser la cota $1.200 \mathrm{~m}$, por encima de la cual se encontrarán las cabeceras con mayor pendiente y procesos de conexión con vertientes activas (canchales, debris flow, etc.).

Se estudió la posibilidad de añadir el factor pendiente para matizar la altitud, pero aportaba poco y, por otro lado, es un factor con un fiel reflejo en la geomorfología del cauce. El tamaño de cuenca nos pareció desde el primer momento un factor poco relevante por solapamiento, ya que tiene un fiel reflejo en el caudal. En cuanto a los factores latitud, longitud y distancia desde nacimiento, los hemos considerado caracterizadores, pero no tipificadores.

En suma, se proponen tres tipos en función de la topografía:

A1) Cursos de alta montaña, por encima de $1.200 \mathrm{~m}$.

A2) Cursos de montaña media, entre 800 y $1.200 \mathrm{~m}$.

A3) Cursos medios y bajos, por debajo de $800 \mathrm{~m}$.

\subsubsection{Factores hidroclimáticos}

Entre todos ellos el caudal es, con mucho, el más relevante. Además, los otros criterios deben ser desechados: temperatura y precipitaciones en la cuenca porque van a tener una respuesta en forma de caudal, y capacidad de neutralización de ácidos y cloruros porque no se dispone de datos suficientes y sería preciso un trabajo 
de campo excesivamente costoso para los resultados a obtener. Ahora bien, más interesante como criterio que el caudal absoluto parece el caudal específico $\left(1 / \mathrm{s} / \mathrm{km}^{2}\right)$, ya que da una idea del déficit hídrico de cada cuenca. Además de estos cursos definidos por su caudal específico se ha añadido un cuarto tipo para los de caudal esporádico.

Q1) Cursos de caudal alto, con un caudal específico superior a $15 \mathrm{l} / \mathrm{s} / \mathrm{km}^{2}$

Q2) Cursos de caudal medio, con caudal específico entre 5 y $15 \mathrm{l} / \mathrm{s} / \mathrm{km}^{2}$

Q3) Cursos de caudal bajo, con un caudal específico inferior a $5 \mathrm{l} / \mathrm{s} / \mathrm{km}^{2}$

Q4) Torrentes y ramblas de caudal esporádico

Q5) Cursos de caudal y régimen hidrológico muy alterados antrópicamente, que han sido reclasificados como masas de agua artificiales o muy modificadas

Otros descriptores del funcionamiento hidrológico podrían ser empleados como criterios, como por ejemplo el régimen estacional y la irregularidad interanual, ya que son indicadores fundamentales y parámetros con gran valor comparativo y diferenciador entre cuencas, incluso si se encuentran próximas. Sin embargo, sólo disponemos de datos reales en las estaciones de aforo, por lo que en el resto de la red hay que estimarlos. Además, resultarían muchos tipos, debido a la diversidad de casos. Ahora bien, en Aragón se registra una división muy clara entre dos funcionamientos hidroclimáticos generales:

P) Cursos de funcionamiento hidroclimático pirenaico, con caudales altos invernales-primaverales y escasa irregularidad interanual.

1) Cursos de funcionamiento hidroclimático ibérico, con caudales altos otonalesinvernales y fuerte irregularidad interanual, así como intermensual por los profundos estiajes.

En la tipificación realizada se ha priorizado este criterio a la hora de diferenciar los tipos, mientras el caudal específico se ha empleado para establecer subtipos.

\subsubsection{Factores geomorfológicos}

Entre los que aparecen en los sistemas A y B de la Directiva nos parecen fundamentales las morfologías del valle y del cauce, ya que son resultado de toda la dinámica hidromorfológica pretérita y actual y, por tanto, fieles indicadores del funciona- 
miento del curso fluvial como sistema. En cambio, los restantes factores que propone la Directiva no son útiles para una tipificación básica. Así, los factores geología y composición media del sustrato podrían servir, en todo caso, para establecer subtipos en casos muy claros de cauces primarios. Pero los principales ríos han recorrido tal diversidad de terrenos que no pueden clasificarse en función de dichos parámetros. Los factores energía del flujo, anchura, profundidad y transporte de sólidos son muy interesantes en la caracterización y útiles para valorar la dinámica actual del sistema, pero requieren un arduo trabajo de campo que los hace inconvenientes para esta fase de tipificación.

Las tipologías de cauces y valles son muy variadas, por lo que es precisa una notable simplificación. Para lograr la misma se ha pensado en las consecuencias bióticas, es decir, se ha observado especialmente en qué medida cauce y valle permiten el desarrollo de un corredor ribereño. La geomorfología del cauce -para algunos autores el "estilo fluvial"- es un indicador fundamental del funcionamiento de todo el sistema fluvial, ya que representa la respuesta a la circulación de materia y energía por la red de drenaje. Para la diferenciación definitiva de estos tipos es imprescindible el trabajo de campo, pero en una tipificación básica debe ser suficiente con su comprobación en fotografías aéreas. Pueden distinguirse cinco grandes tipologías y en algunos casos diversas variantes:

G1) Cursos encajados, que prácticamente carecen de corredor ribereño por la estrechez del fondo de valle.

G1-1) Cursos encajados en $V$, es decir, en valles de perfil transversal en forma de $V$.

G1-2) Cursos encajados en cañón, es decir, en profundos valles de paredes verticales o próximas a la verticalidad.

G2) Cursos con ribera (de menos de $300 \mathrm{~m}$ de anchura) en valles moderadamente abiertos.

G2-1) Cauces poco sinuosos, con índice de sinuosidad inferior a 1,25.

G2-2) Cauces meandriformes, con índice de sinuosidad superior a $1,25 \mathrm{y}$ escaso material grueso.

G2-3) Cauces meandriformes con barras, con índice de sinuosidad superior a 1,25 y abundante material grueso en barras laterales.

G2-4) Cauces trenzados, con subdivisión en islas y brazos, con abundante material grueso.

G2-5) Cursos de ribera eliminada o muy constreñida por ocupación antrópica. 
G3) Cursos con extenso corredor ribereño (más de $300 \mathrm{~m}$ de anchura) en un amplio llano de inundación dentro de anchos valles en artesa.

G3-1) Cauces trenzados muy extensos.

G3-2) Cauces de transición a meandriformes muy extensos, con barras e islas en un corredor ribereño marcadamente sinuoso-meandriforme.

G3-3) Cursos meandriformes libres, que divagan sobre un extenso llano de inundación.

G4) Artesas glaciares, valles de fondo plano y paredes escarpadas

G4-1) Cauces meandriformes o anastomosados, con dominio de material fino.

G4-2) Cauces trenzados, con abundante material grueso.

G5) Ramblas de llano de inundación encajado y cauce complejo, así como otras morfologías menores (tollos, surcos, rills...).

G6) Cauces de morfología totalmente alterada por embalsamiento (G6-1) o canalización (G6-2). Los casos que responden a este tipo se han reclasificado como masas de agua artificiales o muy modificadas.

\section{Tipificación básica}

Así pues, definitivamente se han elegido como criterios de tipificación la altitud, el funcionamiento hidroclimático general, el caudal específico y la geomorfología del cauce y del valle. A partir de la combinación de los criterios seleccionados se han establecido 12 tipos y 79 subtipos, tal como se especifica en el cuadro 4.

Habría que añadir un tipo 13 para los cursos de agua muy modificados, que contarían con los siguientes subtipos:

G6-1) Embalsados

G6-2) Canalizados

Q5) De caudal y régimen hidrológico muy alterados antrópicamente

G6-2.Q5) Canalizados y con caudal y régimen hidrológico muy alterados antrópicamente 
Cuadro 4. Tipos y subtipos resultantes del proceso metodológico y aplicables a los cursos fluviales de Aragón.

\begin{tabular}{|c|c|c|c|c|c|}
\hline \multicolumn{3}{|r|}{ TIPO } & \multicolumn{3}{|c|}{ SUBTIPO } \\
\hline $\mathrm{N}^{\circ}$ & CÓDIGO & DENOMINACIÓN & \multicolumn{2}{|c|}{ DENOMINACION } & CÓngo \\
\hline 1 & A1.P.Q1 & $\begin{array}{c}\text { CURSOS DE ALTA } \\
\text { MONTAÑA PIRENAICA }\end{array}$ & $\begin{array}{l}\text { Encajados en } \mathrm{V} \\
\text { Encajados en cañón } \\
\text { Artesas cauce meandriforme } \\
\text { Artesas con cauce trenzado }\end{array}$ & $\begin{array}{l}\text { con caudal alto o medio } \\
\text { torrentes } \\
\text { con caudal alto o medio } \\
\text { con caudal alto o medio } \\
\text { con caudal alto o medio }\end{array}$ & $\begin{array}{l}\text { A1.P.G1-1.Q1-2 } \\
\text { A1.P.G1-1.Q4 } \\
\text { A1.P.G1-2.Q1-2 } \\
\text { A1.P.G4-1.Q1-2 } \\
\text { A1.P.G4-2.Q1-2 }\end{array}$ \\
\hline 2 & A2.P.G1 & $\begin{array}{c}\text { CURSOS DE MONTAÑA } \\
\text { MEDIA PIRENAICA } \\
\text { ENCAJADOS }\end{array}$ & $\begin{array}{l}\text { Encajados en V } \\
\text { Encajados en cañón }\end{array}$ & $\begin{array}{l}\text { con caudal alto } \\
\text { con caudal medio } \\
\text { con caudal bajo } \\
\text { torrentes } \\
\text { con caudal alto } \\
\text { con caudal medio } \\
\text { con caudal bajo }\end{array}$ & $\begin{array}{l}\text { A2.P.G1-1.Q1 } \\
\text { A2.P.G1-1.Q2 } \\
\text { A2.P.G1-1.Q3 } \\
\text { A2.P.G1-1.Q4 } \\
\text { A2.P.G1-2.Q1 } \\
\text { A2.P.G1-2.Q2 } \\
\text { A2.P.G1-2.Q3 }\end{array}$ \\
\hline 3 & A2.P.G2 & $\begin{array}{c}\text { CURSOS DE MONTAÑA } \\
\text { MEDIA PIRENAICA } \\
\text { CON RIBERA }\end{array}$ & $\begin{array}{l}\text { Poco sinuosos } \\
\text { Meandriformes } \\
\text { Meandriformes con barras } \\
\text { Trenzados } \\
\text { Ribera eliminada }\end{array}$ & $\begin{array}{l}\text { con caudal alto } \\
\text { con caudal medio o bajo } \\
\text { con caudal alto } \\
\text { con caudal medio o bajo } \\
\text { con caudal alto } \\
\text { con caudal medio o bajo } \\
\text { con caudal alto } \\
\text { con caudal medio o bajo } \\
\text { con caudal alto } \\
\text { con caudal medio o bajo }\end{array}$ & $\begin{array}{l}\text { A2.P.G2-1.Q1 } \\
\text { A2.P.G2-1.Q2-3 } \\
\text { A2.P.G2-2.Q1 } \\
\text { A2.P.G2-2.Q2-3 } \\
\text { A2.P.G2-3.Q1 } \\
\text { A2.P.G2-3.Q2-3 } \\
\text { A2.P.G2-4.Q1 } \\
\text { A2.P.G2-4.Q2-3 } \\
\text { A2.P.G2-5.Q1 } \\
\text { A2.P.G2-5.Q2-3 }\end{array}$ \\
\hline 4 & A2.P.G3 & $\begin{array}{c}\text { CURSOS DE MONTAÑA } \\
\text { MEDIA PIRENAICA } \\
\text { CON CORREDOR } \\
\text { RIBEREÑO EXTENSO }\end{array}$ & $\begin{array}{l}\text { Trenzados } \\
\text { De transición a meandriforme }\end{array}$ & $\begin{array}{l}\text { con caudal alto } \\
\text { con caudal alto } \\
\text { con caudal medio o bajo }\end{array}$ & $\begin{array}{l}\text { A2.P.G3-1.Q1 } \\
\text { A2.P.G3-2.Q1 } \\
\text { A2.P.G3-2.Q2 }\end{array}$ \\
\hline 5 & A2.I.G1 & $\begin{array}{c}\text { CURSOS DE MONTAÑA } \\
\text { MEDIA IBÉRICA } \\
\text { ENCAJADOS }\end{array}$ & $\begin{array}{l}\text { Encajados en V } \\
\text { Encajados en cañón }\end{array}$ & $\begin{array}{l}\text { con caudal medio } \\
\text { con caudal bajo } \\
\text { torrentes } \\
\text { con caudal medio } \\
\text { con caudal bajo }\end{array}$ & $\begin{array}{l}\text { A2.I.G1-1.Q2 } \\
\text { A2.I.G1-1.Q3 } \\
\text { A2.I.G1-1.Q4 } \\
\text { A2.I.G1-2.Q2 } \\
\text { A2.I.G1-2.Q3 }\end{array}$ \\
\hline 6 & A2.I.G2 & $\begin{array}{c}\text { CURSOS DE MONTANA } \\
\text { MEDIA IBÉRICA } \\
\text { CON RIBERA }\end{array}$ & $\begin{array}{l}\text { Poco sinuosos } \\
\text { Meandriformes } \\
\text { Meandriformes con barras } \\
\text { Trenzados } \\
\text { Ribera eliminada }\end{array}$ & $\begin{array}{l}\text { con caudal medio } \\
\text { con caudal bajo } \\
\text { con caudal medio } \\
\text { con caudal bajo } \\
\text { con caudal medio } \\
\text { con caudal bajo } \\
\text { con caudal medio } \\
\text { con caudal bajo } \\
\text { con caudal medio } \\
\text { con caudal bajo }\end{array}$ & $\begin{array}{l}\text { A2.I.G2-1.Q2 } \\
\text { A2.I.G2-1.Q3 } \\
\text { A2.I.G2-2.Q2 } \\
\text { A2.I.G2-2.Q3 } \\
\text { A2.I.G2-3.Q2 } \\
\text { A2.I.G2-3.Q3 } \\
\text { A2.I.G2-4.Q2 } \\
\text { A2.I.G2-4.Q3 } \\
\text { A2.I.G2-5.Q2 } \\
\text { A2.I.G2-5.Q3 }\end{array}$ \\
\hline
\end{tabular}




\begin{tabular}{|c|c|c|c|c|c|}
\hline 7 & A3.P.G1 & $\begin{array}{c}\text { CURSOS MEDIOS Y BAJOS } \\
\text { PIRENAICOS ENCAJADOS }\end{array}$ & $\begin{array}{l}\text { Encajados en V } \\
\text { Encajados en cañón }\end{array}$ & $\begin{array}{l}\text { con caudal alto } \\
\text { con caudal medio } \\
\text { con caudal bajo } \\
\text { con caudal alto } \\
\text { con caudal medio } \\
\text { con caudal bajo }\end{array}$ & $\begin{array}{l}\text { A3.P.G1-1.Q1 } \\
\text { A3.P.G1-1.Q2 } \\
\text { A3.P.G1-1.Q3 } \\
\text { A3.P.G1-2.Q1 } \\
\text { A3.P.G1-2.Q2 } \\
\text { A3.P.G1-2.Q3 }\end{array}$ \\
\hline 8 & A3.P.G2 & $\begin{array}{c}\text { CURSOS MEDIOS Y BAJOS } \\
\text { PIRENAICOS CON RIBERA }\end{array}$ & $\begin{array}{l}\text { Poco sinuosos } \\
\text { Meandriformes } \\
\text { Meandriformes con barras } \\
\text { Trenzados } \\
\text { Ribera eliminada }\end{array}$ & $\begin{array}{l}\text { con caudal alto } \\
\text { con caudal medio } \\
\text { con caudal bajo } \\
\text { con caudal alto } \\
\text { con caudal medio } \\
\text { con caudal bajo } \\
\text { con caudal alto } \\
\text { con caudal medio } \\
\text { con caudal bajo } \\
\text { con caudal alto } \\
\text { con caudal medio } \\
\text { con caudal bajo } \\
\text { con caudal alto } \\
\text { con caudal medio } \\
\text { con caudal bajo }\end{array}$ & $\begin{array}{l}\text { A3.P.G2-1.Q1 } \\
\text { A3.P.G2-1.Q2 } \\
\text { A3.P.G2-1.Q3 } \\
\text { A3.P.G2-2.Q1 } \\
\text { A3.P.G2-2.Q2 } \\
\text { A3.P.G2-2.Q3 } \\
\text { A3.P.G2-3.Q1 } \\
\text { A3.P.G2-3.Q2 } \\
\text { A3.P.G2-3.Q3 } \\
\text { A3.P.G2-4.Q1 } \\
\text { A3.P.G2-4.Q2 } \\
\text { A3.P.G2-4.Q3 } \\
\text { A3.P.G2-5.Q1 } \\
\text { A3.P.G2-5.Q2 } \\
\text { A3.P.G2-5.Q3 }\end{array}$ \\
\hline 9 & A3.I.G1 & $\begin{array}{l}\text { CURSOS MEDIOS Y BAJOS } \\
\text { IBÉRICOS ENCAJADOS }\end{array}$ & $\begin{array}{l}\text { Encajados en V } \\
\text { Encajados en cañón }\end{array}$ & $\begin{array}{l}\text { con caudal medio } \\
\text { con caudal bajo } \\
\text { con caudal medio } \\
\text { con caudal bajo }\end{array}$ & $\begin{array}{l}\text { A3.I.G1-1.Q2 } \\
\text { A3.I.G1-1.Q3 } \\
\text { A3.I.G1-2.Q2 } \\
\text { A3.I.G1-2.Q3 }\end{array}$ \\
\hline 10 & A3.I.G2 & $\begin{array}{l}\text { CURSOS MEDIOS Y BAJOS } \\
\text { IBÉRICOS CON RIBERA }\end{array}$ & $\begin{array}{l}\text { Poco sinuosos } \\
\text { Meandriformes } \\
\text { Meandriformes con barras } \\
\text { Trenzados } \\
\text { Ribera eliminada }\end{array}$ & $\begin{array}{l}\text { con caudal bajo } \\
\text { con caudal bajo } \\
\text { con caudal bajo } \\
\text { con caudal bajo } \\
\text { con caudal bajo }\end{array}$ & $\begin{array}{l}\text { A3.I.G2-1.Q3 } \\
\text { A3.I.G2-2.Q3 } \\
\text { A3.I.G2-3.Q3 } \\
\text { A3.I.G2-4.Q3 } \\
\text { A3.I.G2-5.Q3 } \\
\end{array}$ \\
\hline 11 & A3.G3 & \begin{tabular}{|} 
CURSOS MEDIOS Y BAJOS \\
CON CORREDOR \\
RIBEREÑO EXTENSO
\end{tabular} & $\begin{array}{l}\text { Trenzados } \\
\text { De transición a meandriforme } \\
\text { Meandriformes libres }\end{array}$ & $\begin{array}{l}\text { con caudal alto } \\
\text { con caudal medio } \\
\text { con caudal bajo } \\
\text { con caudal alto } \\
\text { con caudal medio } \\
\text { con caudal bajo } \\
\text { con caudal medio }\end{array}$ & $\begin{array}{l}\text { A3.G3-1.Q1 } \\
\text { A3.G3-1.Q2 } \\
\text { A3.G3-1.Q3 } \\
\text { A3.G3-2.Q1 } \\
\text { A3.G3-2.Q2 } \\
\text { A3.G3-2.Q3 } \\
\text { A3.G3-3.Q2 }\end{array}$ \\
\hline 12 & G5.Q4 & RAMBLAS & \multicolumn{2}{|c|}{$\begin{array}{l}\text { De montaña media } \\
\text { Asociadas a cursos medios y bajos }\end{array}$} & $\begin{array}{l}\text { G5.Q4.A2 } \\
\text { G5.Q4.A3 }\end{array}$ \\
\hline
\end{tabular}

\section{Consideración final}

La necesidad de elaborar una metodología de trabajo para la tipificación de los sistemas fluviales en Aragón es el objetivo prioritario del presente trabajo. Una vez establecida esta metodología se deberá proceder posteriormente a su desarrollo práctico con la asignación de cada tramo de la red fluvial aragonesa a uno de los tipos defi- 
nidos. Para ello es preciso dividir dicha red en sectores funcionales internamente homogéneos y claramente diferenciados entre sí (Amoros y Petts, 1993). Los criterios básicos para establecer esta tramificación deberían ser pendiente, geomorfología del cauce y del valle (estilo fluvial) y caudal, tanto de acuerdo con la experiencia de los miembros del equipo como según las recomendaciones del Guidance standard CEN TC 230/WG 2/TG 5: N32. Los tramos son la base para establecer puntos de muestreo y para las clasificaciones, análisis y valoraciones posteriores. Esta tipificación, basada fundamentalmente en criterios hidromorfológicos, servirá de base para la definición de tipos fluviales caraterizados posteriormente mediante parámetros ecológicos. En definitiva, esta tipificación será útil tanto para la caracterizaciốn de los cursos fluviales en Aragón, como para la valoración de su actual estado ecológico y su seguimiento en el futuro.

\section{Agradecimientos}

El presente trabajo se inició metodológicamente en el marco del proyecto de investigación PI-27-1999 "Vegetación ripícola y geomorfología fluvial: procesos, modelos de interacción y aplicaciones a la gestión del territorio y a la prevención de riesgos en ríos de Euskal Herria", financiado por el Gobierno Vasco.

En la actualidad el equipo firmante de este artículo está desarrollando la metodología expuesta en los estudios "Determinación del estado ecológico de los ríos del Parque Natural de la Sierra y Cañones de Guara" y "Determinación del estado ecológico de los ríos de Aragón", para el Departamento de Medio Ambiente del Gobierno de Aragón, a cuyos responsables agradecemos la posibilidad de presentar esta publicación.

\section{Bibliografía}

Amoros, C. y Petts, G.E. (1993): Hydrosystèmes fluviaux. Paris, Masson, 300 pp.

Boon, P.J., Wilkinson, J. y Martin, J. (1998): "The application of SERCON (System for Evaluating Rivers for Conservation) to a selection of rivers in Britain". Aquatic Conservation.
Marine and Fresbwater Ecosystems, 8 (4), p. 597-616.

Brice, J.C. (1984): "Planform properties of meandering rivers". In Elliott, C.M. (ed.): River meandering: proceedings of the conference Rivers '83, Nueva York, American Sociery of Civil Engineers, p. 1-14. 
Brice, J.C. y Blodgett, J.C. (1978): Counter measures for bydraulic problems at bridges. Analysis and Assessment. Washington, FHWA-RD-78-162, vol. 1.

Brierley, G. y Fryirs, K. (2000): "River Styles, a geomorphic approach to catchment characterisation: implications for river rehabilitation in Bega catchment, New South Wales, Australia". Environmental Management, 25 (6), p. 661-679.

Brierley, G., Fryirs, K., Outhet, D. y Massey, C. (2002): "Application of the River Styles framework as a basis for river management in New South Wales, Australia". Applied Geography, 22, p. 91-122.

Church, M. y Rood, K. (1983): Catalogue of alluvial river cbannel regime data. Ottawa. Natural Sciences and Engineering Research Council of Canada.

Commission of the European Communities (2002): A guidance standard for assessing the bydromorphological features of rivers. CEN TC 230/WG 2/TG 5: N32.

Downs, P.W. (1995): "River channel classification for channel management purposes". In Gurnell, A.M. y Petts, G.E. (eds.): Changing river cbannels, John Wiley and sons, p. 347-365.

Federal Interagency Stream Restoration Working Group (1998): Stream corridor restoration: principles, processes and practices. Washington, F.I.S.R.W.G.

Fryirs, K. y Brierley, G. (2001): "A geomorphic approach to the identification of river recovery potential". Pbysical Geograpby, 21(3), p. 244-277.

Galay, V.J., Kellerhals, R. y Bray, D.I. (1973): "Diversity of river types in Canada". En Fluvial process and sedimentation, Procedings of Hydrology Sympossium. National Research Council of Canada, p. 217-250.

Jarrett, R.D. (1987): "Errors in slope-area computations of peak discharges in mountain streams". Journal of Hydrology, 96, p. 53-67.

Kellerhals, R., Neill, C.R. y Bray, D.I. (1972): Hydraulic and geomonphic characteristics of rivers in Alberta. Research Council of Alberta. River Engineering and Surface Hydrology Report 72-1, 52 pp.

Kellerhals, R., Church, M. y Bray, D.I. (1976): "Classification and analysis of river processes". Journal of the Hydraulics Division, 102, p. 813-829.

Kellerhals, R. y Church, M. (1989): "The morphology of large rivers: characterization and management". In Dodge, D.P. (ed.): Proceedings of the International Large River Symposium. Canadian Spec. Publ. Fish. Aquat. Sci, 106, p. 31-48.

Knighton, D. (1998): Fluvial forms and processes. A new perspective. London, Arnold, $383 \mathrm{pp}$.

Ladson, A.R. et al. (1999): "Development and testing of an Index of Stream Condition for waterway management in Australia". Freshwater Biology, 41 (2), p. 453-468.

Lane, E.W. (1957): A study of the shape of channels formed by natural streams flowing in erodible material. Omaha, Missouri River Division Sediment Series, 9.

Lane, S. (1995): "The dynamics of dynamic river channels". Geograpby, 80 (2), p. 147-162.

Leopold, L.B. y Wolman, M.G. (1957): "River channel pattern: braided, meandering and 
straight". U.S. Geological Survey, prof. paper 282B, p. 39-85.

Mollard, J.D. (1973): "Air-photo interpretation of fluvial features". Fluvial process and sedimentation. Procedings of Hydrology Symposium National Research Council of Canada, p. 341-380.

Mosley, M.P. (1987): "The classification and characterization of rivers". In Richards, K.S. (ed.): River channels: environmental processes, p. 295-320.

Nanson, G.C. y Croke, J.C. (1992): "A genetic classification of floodplains". Geomonphology, 4, p. $459-486$.

Ollero, A. (2000): "Primera aproximación a una clasificación de cursos fluviales aplicable a la ordenación". Lurralde, 23, p. $125-133$.

Ollero, A. (2000): "Los paisajes fluviales: modelo de análisis y propuestas de ordenación, con aplicación al río Ara". Sobrarbe, 6, p. 99-132.

Ollero, A. (2002): "La Geografía Física y la Geomorfología en el estudio de los sistemas fluviales: dinámica fluvial, riesgos y ordenación". En Sáinz, J.A. y Garmendia, C. (eds.): Ordenación de âreas fluviales en el Norte de España. Santander, Univ. de Cantabria, p. 37-59.

Olsen, A. (2001): "The new Water Framework Directive for the European Union. Main principles and obligations. Prospects for a sustainable water policy for the coming decades". In Grande, N., Arrojo, P. y Martínez Gil, J. (coords.): Una cita europea con la nueva cultura del agua: la Directiva Marco. Perspectivas en Portugal y España. Zaragoza, Univ. de Zaragoza e Institución Fernando el Católico, p. 17-30.
Prat, N. (2001): "Problemas y perspectivas en la definición del estado ecológicó de los ecosistemas fluviales peninsulares ibéricos". En Grande, N., Arrojo, P. y Martínez Gil, J. (coords.): Una cita europea con la nueva cultura del agua: la Directiva Marco. II Congreso Ibérico sobre Planificación y Gestión de Aguas, Zaragoza, Univ. de Zaragoza e Institución Fernando el Católico. p. 39-54.

Prat, N. y Munné, A. (1999): Regionalización de la cuenca del Ebro. Zaragoza, Confederación Hidrográfica del Ebro.

Raven, P.J., Holmes, N.T.H., Dawson, F.H. y Everard, M. (1998): "Quality assessment using River Habitat Survey data". Aquatic Conservation. Marine and Freshwater Ecosystems, 8 (4), p. 477-499.

Rosgen, D.L. (1994): "A classification of natural rivers". Catena, 22 (3), p. 169-199.

Rosgen, D.L. (1996): Applied river morpho$\log y$. Wildland Hydrology.

Roussel, P. (dir.) (1999): Les outils d'évaluation de la qualité des cours d'eau (S.E.Q.). Principes généraux. Paris, Agences de l'Eau, 12 pp.

Rust, B.R. (1978): "A classification of alluvial channel systems". In Miall, A.D. (ed.): Fhuvial Sedimentology, Mem. Canadian Society Petroleum Geologists, 5, p. 187-198.

Schmitt, L. (2001): Typologie bydro-géomorphologique fonctionelle des cours d'eau. Recherche méthodologique appliquée aux systèmes fluviaux d'Alsace. Estrasburgo, Université Louis Pasteur, tesis doctoral inédita.

Schumm, S.A. (1963): A tentative classification of alluvial river channels. U.S. Geological Survey, circular 477 . 
Schumm, S.A. (1977): The fluvial system. New York, Wiley, 338 pp.

Tartar, P. (2001): Mise en oeuvre $d u$ système d'évaluation de la qualité physique des cours d'eau sur l'Andelle. Note de syntbèse. Rouen DIREN HauteNormandie.

Thorne, C.R. (1998): Stream reconnaissance bandbook: geomorpbological investigation and analysis of river channels. Chichester, Wiley, $133 \mathrm{pp}$

Ureña, J.M. y Ollero, A. (2000): "Criterios y propuestas para la ordenación de áreas fluviales". Ciudad y territorio, Estudios Territoriales, XXXII (126), p. 689-710.
Wallin, M., Wiederholm, T. y Johnson, R.K. (2002): Guidance on establisbing reference conditions and ecological status class boundaries for inland surface waters. Water Framework Directive Common Implementation Strategy Working Group 2.3 REFCOND, 80 pp., CIS-WFD

Wilkinson, J., Martin, J., Boon, P.J. \& Holmes, N.T.H. (1998): "Convergence of field survey protocols for SERCON (System for Evaluating Rivers for Conservation) and RHS (River Habitat Survey)". Aquatic Conservation. Marine and Fresbwater Ecosystems, 8 (4), p. 579-596.

Williams, G.P. (1978): "Bank-full discharge of rivers". Water Resources Research, 14, p. 1141-1154. 